\title{
Investigation of Two-Dimensional Viscoelastic Fluid with Nonuniform Heat Generation over Permeable Stretching Sheet with Slip Condition
}

\author{
Haroon Ur Rasheed $\left(D,{ }^{1}\right.$ Zeeshan Khan, ${ }^{1}$ Saeed Islam, ${ }^{2}$ Ilyas Khan $\left(\mathbb{D},{ }^{3}\right.$ Juan L. G. Guirao ${ }^{1},{ }^{4}$ \\ and Waris Khan ${ }^{5}$ \\ ${ }^{1}$ Sarhad University of Science and Information Technology, Peshawar, KPK 25000, Pakistan \\ ${ }^{2}$ Department of Mathematics, Abdul Wali Khan University Mardan, Mardan, KPK 23000, Pakistan \\ ${ }^{3}$ Faculty of Mathematics and Statistics, Ton Duc Thang University, Ho Chi Minh City 72915, Vietnam \\ ${ }^{4}$ Departmento de Matematica Aplicada y Estadistica, Universidad Politecnica de Cartagena, Hospital de Marina, \\ 30203 Cartagena, Region de Murcia, Spain \\ ${ }^{5}$ Institute of Numerical Sciences, Kohat University of Science and Technology, Kohat, Pakistan \\ Correspondence should be addressed to Ilyas Khan; ilyaskhan@tdtu.edu.vn
}

Received 20 July 2019; Revised 9 October 2019; Accepted 30 October 2019; Published 4 December 2019

Academic Editor: Dimitri Volchenkov

Copyright (C) 2019 Haroon Ur Rasheed et al. This is an open access article distributed under the Creative Commons Attribution License, which permits unrestricted use, distribution, and reproduction in any medium, provided the original work is properly cited.

\begin{abstract}
Here, in this research article, we have investigated an incompressible viscoelastic fluid flow over a uniform stretching surface sheet along with slip boundary conditions in the presence of porous media. The partial differential equations which govern the fluid flow are changed into ordinary differential equations through suitable similarity transformation variables. Finally, the transformed ordinary differential equations are solved with the help of a seminumerical technique known as the homotopy analysis method (HAM). The uniqueness of our study is not only to analyze and carry out the effect of the elastic parameter but also to account for viscous dissipation which is important in the case of optically transparent flow. The novel effects for the parameters which affect the flow and heat transfer, such as the Eckert number, porous medium parameter, and the velocity slip parameter, are studied through graphs. Also, the convergence analysis for the proposed method is addressed. Additionally, for the sake of validation, the present work is also compared with the already published work and an outstanding agreement is found.
\end{abstract}

\section{Introduction}

Owing to the importance of the fluid flow over a stretching surface, it, has resulted in active studies, due to its practical applications such as hot rolling, fiber plating, and lubrication porous. Crane [1] first introduced an analytical solution of Newtonian boundary layer flow due to a stretching surface. Vleggar [2] studied the laminar flow of Newtonian fluid on a continuous accelerating stretching surface. Dutta et al. [3] investigated the temperature field flow due to a stretching sheet with uniform heat flux. In the content, a similar problem of Newtonian fluid flow due to the stretching surface has been investigated by many researchers $[4,5]$.

Investigation of a viscoelastic fluid over a continuous stretching surface finds many important applications in the fields of engineering fluid mechanics such as inks, paints, jet fuels, polymer extrusion, drawing of plastic fiber, and wire. The over increasing applications to this type of fluid, many researchers turned to the study of this type under different situations. Vajravelu and Rollins [6] studied viscoelastic fluid over a stretching surface with the effect of heat transfer. Andersson [7] analyzed the effect of MHD on viscoelastic fluid flow due to the stretching surface. Incompressible flow 
of viscoelastic fluid and heat transfer over a stretching sheet embedded in a porous medium have been investigated by Suhas and Veena [8]. Viscoelastic boundary layer fluid flow and heat transfer over an exponential stretching sheet have been discussed by Sanjaya and Khan [9]. Nandeppanavar [10] studied the flow and heat transfer characteristic of a viscoelastic fluid over an impermeable stretching sheet embedded in a porous medium with viscous dissipation and heat transfer.

In the above studies, the effect of velocity slip is absent. This phenomenon is very important in fluid mechanics. It was first introduced by Navier [11]. Thompson and Troian studied [12] the incompressible flow at the solid surface with general boundary conditions. Slip effects and heat transfer analysis in a viscous fluid over an oscillatory stretching surface have been studied by Abbas et al. [13]. MHD slip flow of viscoelastic fluid over the stretching surface has been investigated by Turkyilmazoglu [14]. Ferrás et al. [15] analyzed the slip flow of Newtonian and viscoelastic fluids. The effect of slip and MHD on viscoelastic convection flow in a vertical channel has been discussed by Singh [16]. Krishan [17] analyzed magnetohydrodynamic mixed convection viscoelastic slip flow through a porous medium in a vertical porous channel with thermal radiation. The effect of slip conditions on the peristaltic flow of a Jeffrey fluid with a Newtonian fluid is studied by Vajravelu et al. [18].

For the non-Newtonian fluids, the perdition of heat transfer analysis is very important due to its practical engineering uses, such as food processing, flow through filtering media, and oil recovery. Because of the above motivation, in the present work, a new visualization for the effects of the nonuniform heat generation/ absorption, velocity slip, and viscous dissipation with heat transfer flow of viscoelastic fluid due to the stretching surface embedded in a porous medium is analyzed. Recently, viscoelastic Oldroyd 8 -constant fluid has been analyzed for wire coating by Khan et al. [19] using the Runge-Kutta method with heat transfer effect. Prasad et al. [20] investigated magnetohydrodynamic mixed convection heat flow over a nonlinear sheet with temperature-dependent viscosity. Similarly, Awati [21] carried out an analysis of MHD viscous flow with a heat source. Series and analytical solutions have been obtained, and the effects of emerging parameters were discussed through graphs. Ahmad et al. [22] investigated a steady flow of a power-law fluid through an artery with a stenosis, and the effects of various parameters of interest were discussed through graphs. A detailed analysis of MHD flow and heat transfer through viscoelastic fluid in the presence of a porous medium in wire coating analysis has been carried out by Khan et al. [23].

In the present study, the two-dimensional flow of viscoelastic fluid with nonuniform heat source generation along a permeable stretching sheet is investigated analytically by semianalytical method (HAM) with slip conditions. The modeled partial differential equations are converted to ordinary differential equations by using similarity variables. The series solutions have been obtained by HAM. The effect of emerging parameters involved in the solution has been discussed through graphs in detail. Additionally, for the accuracy of the results, the present work is also compared with the published work of Rajagopal et al. [24].

\section{Formulation of the Problem}

In this section, we will consider a two-dimensional boundary layer flow of an incompressible viscoelastic fluid over a stretching sheet embedded in a porous medium. The origin is located at a slit, through which the sheet (see Figure 1) is drawn through the fluid medium. The $x$-axis is chosen along the sheet and the $y$-axis is taken normal to it. The sheet is assumed to have the velocity $u=c x$, where $x$ is the coordinate measured along the stretching surface and $C>0$ is a constant for a stretching sheet. Likewise, the temperature distribution for the sheet is assumed to be in the form $T_{w}=T_{\infty}+A x^{r}$, where $T_{w}$ is the temperature of the sheet, $T_{\infty}$ is the temperature of the ambient, and $A$ and $r$ are constants. Also, the sheet is assumed to be porous with the suction velocity $v_{w}$. Making the usual boundary layer approximations, the boundary layer equations read

$$
\begin{aligned}
\frac{\partial u}{\partial x}+\frac{\partial v}{\partial y}= & 0 \\
u \frac{\partial u}{\partial x}+v \frac{\partial u}{\partial y}= & \frac{\mu}{\rho} \frac{\partial^{2} u}{\partial x^{2}}-\frac{\mu_{e}}{\rho k} u \\
& -\frac{k_{0}}{\rho}\left(u \frac{\partial^{3} u}{\partial x \partial y^{2}}+v \frac{\partial^{3} u}{\partial y^{3}}-\frac{\partial u}{\partial y} \frac{\partial^{2} u}{\partial x \partial y}+\frac{\partial u}{\partial x} \frac{\partial^{2} u}{\partial y^{2}}\right) \\
u \frac{\partial T}{\partial x}+v \frac{\partial T}{\partial y}= & \frac{k}{\rho c_{p}} \frac{\partial^{2} T}{\partial y^{2}}+\frac{k}{\rho c_{p}}\left(\frac{\mu_{e}}{k} u^{2}+u\left(\frac{\partial u}{\partial x}\right)^{2}\right)+\frac{q^{\prime \prime \prime}}{\rho c_{p}}
\end{aligned}
$$

where $u$ and $v$ are the velocity components in the $x$ and $y$ directions, respectively. $\rho$ is the density of the fluid, $\kappa$ is the fluid thermal conductivity, and $K_{0}$ is a positive parameter associated with the viscoelastic fluid. $T$ is the temperature of the fluid, $\mu$ is the fluid viscosity, $\mu_{e}$ is the dynamic viscosity of the fluid due to the flow in the porous medium, $k$ is the permeability of the porous medium, $q^{\prime \prime \prime}$ is the rate of internal heat generation, and $c_{p}$ is the specific heat at constant pressure. We must observe that in the second term of the right-hand side of equation (3), we follow [25-28].

The boundary conditions with the slip condition [18-20] can be written as

$$
\begin{array}{r}
u=U+a\left(\frac{\partial u}{\partial y}-\frac{k_{0}}{\mu}\left(u \frac{\partial^{2} u}{\partial x \partial y}+v \frac{\partial^{2} u}{\partial y^{2}}+2 \frac{\partial u}{\partial x} \frac{\partial u}{\partial y}\right)\right), \\
v=-v_{w}, T_{w}=T_{\infty}+A x^{r} \text { at } y=0, \\
u \longrightarrow 0, T_{w} \longrightarrow T_{\infty}, \text { as } y \longrightarrow \infty,
\end{array}
$$

where $a$ is the velocity slip factor; the mathematical analysis of the problem is simplified by introducing the following dimensionless coordinates: 


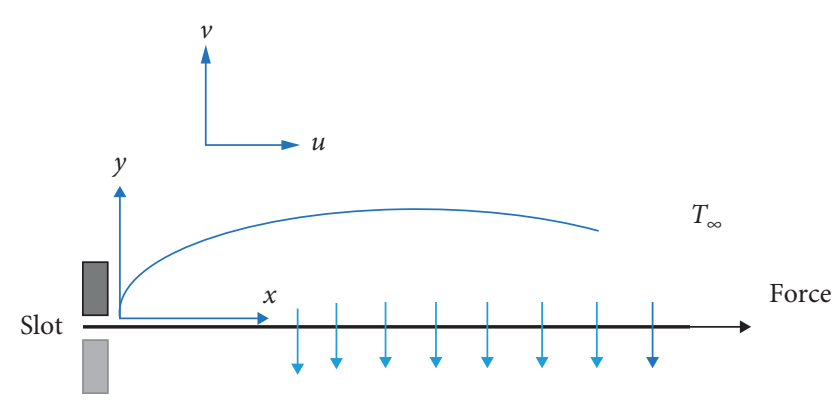

Figure 1: Physical configuration diagram.

$$
\begin{gathered}
\eta=y \sqrt{\frac{c}{v}}, \\
u=c x f^{\prime}(\eta), \\
v=-\sqrt{c v} f(\eta), \\
\theta(\eta)=\left(\frac{T-T_{\infty}}{T_{w}-T_{\infty}}\right),
\end{gathered}
$$

where $\eta$ is the similarity variable, $f(\eta)$ is the dimensionless stream function, $v=\mu / \rho$ is the kinematic viscosity, and $\theta(\eta)$ is the dimensionless temperature. It can be seen that a similarity solution exists only when we take $r=2$. Likewise, the internal heat generation or absorption $q^{\prime \prime \prime}$ is modeled according to the following formula [29]:

$$
q^{\prime \prime \prime}=\left(\frac{k U}{v x}\right)\left[a^{*}\left(T-T_{\infty}\right) e^{-\eta}+b^{*}\left(T_{w}-T_{\infty}\right)\right] .
$$

Therefore, upon using these variables, the boundary layer governing equations (1)-(3) can be written in the following nondimensional form:

$$
f^{\prime \prime \prime}-\left(f^{\prime}\right)^{2}+f f^{\prime \prime}-\beta f^{\prime}+k\left(\left(f^{\prime \prime}\right)^{2}-2 f^{\prime} f^{\prime \prime \prime}+f f^{\prime \prime \prime \prime}\right)=0
$$

$$
\begin{gathered}
\frac{1}{\operatorname{Pr}} \theta^{\prime \prime}+f \theta^{\prime}-2 f^{\prime} \theta+\operatorname{Ec}\left(\beta\left(f^{\prime}\right)^{2}+\left(f^{\prime \prime}\right)^{2}\right) \\
+\frac{1}{\operatorname{Pr}}\left(a^{*} e^{-\eta}+b^{*} \theta\right)=0,
\end{gathered}
$$

and the boundary conditions are as follows:

$$
\begin{aligned}
f=f_{w}, f=1+\lambda\left[\left(1+3 k f^{\prime}\right) f^{\prime \prime}+k f_{w} f^{\prime \prime \prime}\right], & \\
& \theta=1 \text {, at } \eta=\infty,
\end{aligned}
$$

$f^{\prime} \longrightarrow 0, f^{\prime \prime} \longrightarrow 0, \theta \longrightarrow 0$, at $\eta \longrightarrow \infty$

where $\beta=\mu_{e} / \rho c k$ is the porous parameter, $K=c k_{0} / \mu$ is the viscoelastic parameter, $\operatorname{Pr}=\mu c_{p} / \kappa, \kappa$ is the Prandtl number,
$E_{c}=c^{2} / A c_{p}$ is the Eckert number, $f_{w}=v_{w} / \sqrt{c v}>0$ is the suction velocity parameter, and $\lambda=a \sqrt{c / v}$ is the velocity slip parameter.

\section{HAM Solution}

In order to solve equations (7) and (8) under the boundary conditions (9) and (10), we utilize the homotopy analysis method with the following procedure. The solution having the auxiliary parameter $\hbar$ regulates and controls the convergence of the solutions. The initial guesses are selected as follows.

We select the initial approximations such that the boundary conditions are satisfied as follows:

$$
\begin{aligned}
& f_{0}(\eta)=s-1+e^{-\eta} \\
& \theta_{0}(\eta)=e^{-\eta}
\end{aligned}
$$

The linear operators are introduced as $\mathfrak{\Im}_{f}$ and $\mathfrak{\Im}_{\theta}$ :

$$
\begin{gathered}
\mathfrak{\Im}_{f}(f)=f^{\prime \prime \prime}, \\
\mathfrak{\Im}_{\theta}(\theta)=\theta^{\prime \prime} .
\end{gathered}
$$

With the following properties,

$$
\begin{aligned}
\mathfrak{\Im}_{f}\left(c_{1}+c_{2} \eta+c_{3} \eta^{2}+c_{4} e^{-\eta}\right) & =0, \\
\mathfrak{J}_{\theta}\left(c_{5}+c_{6} e^{-\eta}\right) & =0,
\end{aligned}
$$

where $c_{i}(i=1-6)$ are the arbitrary constants in general solution.

The nonlinear operators, according to (7) and (8), are defined as follows:

$$
\begin{aligned}
\aleph_{f}[f(\eta ; p)]= & \frac{\partial^{3} f(\eta ; p)}{\partial \eta^{3}}-\left(\frac{\partial f(\eta ; p)}{\partial \eta}\right)^{2} \\
& +f(\eta ; p) \frac{\partial^{2} f(\eta ; p)}{\partial^{2} \eta}-\beta \frac{\partial f(\eta ; p)}{\partial \eta} \\
& +\kappa\left(\left(\frac{\partial^{2} f(\eta ; p)}{\partial^{2} \eta}\right)^{2}-2 \frac{\partial f(\eta ; p)}{\partial \eta} \frac{\partial^{3} f(\eta ; p)}{\partial \eta^{3}}\right. \\
& \left.+f(\eta ; p) \frac{\partial^{3} f(\eta ; p)}{\partial \eta^{3}}\right)=0, \\
\aleph_{\theta}[f(\eta ; p), \theta(\eta ; p)]= & \frac{1}{\operatorname{Pr}} \frac{\partial^{2} \theta(\eta ; p)}{\partial \eta^{2}}+\operatorname{Pr} f(\eta ; p) \frac{\partial \theta(\eta ; p)}{\partial \eta} \\
& -2 f(\eta ; p) \frac{\partial \theta(\eta ; p)}{\partial \eta} \\
& +E_{c}\left(\beta\left(\frac{\partial \theta(\eta ; p)}{\partial \eta}\right)^{2}+\left(\frac{\partial^{2} \theta(\eta ; p)}{\partial \eta^{2}}\right)^{2}\right) \\
& +\frac{1}{\operatorname{Pr}}\left(a^{*} e^{-\eta}+b^{*} \theta\right)=0 .
\end{aligned}
$$


The auxiliary function become

$$
\mathrm{H}_{f}(\eta)=\mathrm{H}_{\theta}(\eta)=e^{-\eta}
$$

The symbolic software Mathematica is employed to solve $i$ th order deformation equations:

$$
\begin{aligned}
\mathfrak{\Im}_{f}\left[f_{i}(\eta)-\chi_{i} f_{i-1}(\eta)\right] & =\hbar_{f} \mathscr{H}_{f}[f(\eta)] R_{f, i}(\eta), \\
\mathfrak{\Im}_{\theta}\left[\theta_{i}(\eta)-\chi_{i} \theta_{i-1}(\eta)\right] & =\hbar_{\theta} \mathscr{H}_{\theta}(\eta) R_{\theta, i},
\end{aligned}
$$

where $\hbar$ is auxiliary nonzero parameter and

$$
\begin{aligned}
R_{f, i}(\eta)= & f_{m-1}^{\prime \prime \prime}-\sum_{k=0}^{m-1} f_{m-1-k}^{\prime} f_{k}^{\prime}+\sum_{k=0}^{m-1} f_{m-1-k} f_{k}^{\prime}-\beta f_{m-1}^{\prime} \\
& +k\left(\sum_{k=0}^{m-1} f_{m-1-k}^{\prime} f_{k}^{\prime}-2 f_{m-1}^{\prime} \sum_{k=0}^{m-1} f_{m-1-k}^{\prime} f_{k}^{\prime}\right. \\
& \left.+\sum_{k=0}^{m-1} f_{m-1-k}^{\prime} \sum_{l=0}^{k} f_{k-1}^{\prime} f_{l}^{\prime \prime \prime}\right), \\
R_{\theta, i}(\eta)= & \frac{1}{\operatorname{Pr}} \theta_{m-1}^{\prime \prime}+\sum_{k=0}^{m-1} f_{m-1-k} \theta_{k}^{\prime}-2 \sum_{k=0}^{m-1} \theta_{m-1-k} f_{k}^{\prime} \\
& +E_{c}\left(\beta \sum_{k=0}^{m-1} f_{m-1-k} f_{k}^{\prime}+\sum_{k=0}^{m-1} f_{m-1-k} f_{k}^{\prime \prime}\right) \\
& +\frac{1}{\operatorname{Pr}}\left(a^{*} e^{-\eta}+b^{*} \theta\right), \\
\chi_{i}=\left\{\begin{array}{l}
\text { if } i \leq 1 \\
0, \quad \text { if } i>1
\end{array}\right. & \\
1, \quad &
\end{aligned}
$$

are the involved parameters in HAM theory. For more details about the theory of homotopy analysis method, see [30-40].

3.1. Convergence of the Method. To validate the method, the convergence of the method is also necessary. For this purpose, $h$-curve has been drawn which ensures the convergence of the series solution. The calculations are carried out on a personal computer with $4 \mathrm{~GB}$ RAM and $2.70 \mathrm{GHz}$ CPU. The code is developed using computer software Mathematica [41]. In Figures 2 and 3, h-curves are plotted for 20th order of approximation for velocity and temperature profiles, respectively. These figures clearly show the range for admissible values. $h_{f}$ and $h_{\theta}$ is $-1.5 \leq h_{f} \leq-0.3$ and $-1.7 \leq h_{\theta} \leq-0.3$, respectively.

\section{Results and Discussion}

Two-dimensional non-Newtonian viscoelastic fluid with nonuniform heat generation over a permeable stretching sheet embedded in a porous medium has been investigated. The similarity transformation has been applied to transform

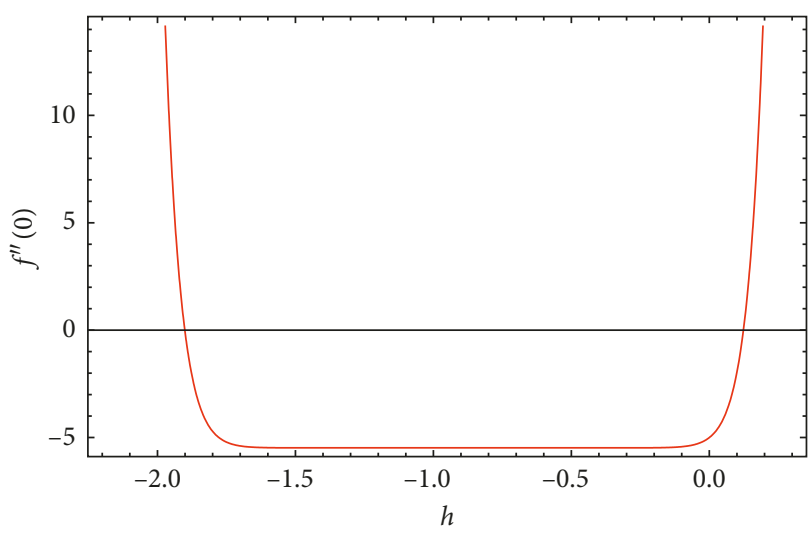

Figure 2: $h$-curve for velocity field.

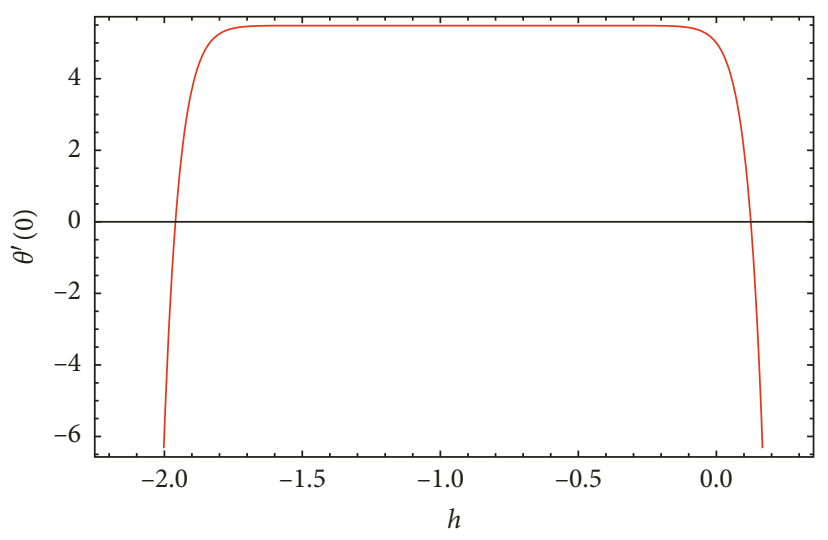

Figure 3: $h$-curve for temperature field.

the PDEs to ODEs. The analytical solution has been obtained by using HAM. For the validation of our analytical solution, a comparison has been done with the published work of Rajagopal et al. [24]. This comparison is given in Table 1 . This ensures that our results are excellent and in agreement with the published work. The computation results are displayed in Figures $4-13$.

From Figure 4, it is observed that the velocity of the fluid decreases with the increasing values of the porous parameter $\beta$. Physically, a greater of beta means a high dynamic viscosity $\mu_{e}$, which corresponds to porous medium and a small permeability for the porous medium, which causes the production of the resistance force to the fluid flow which causes a decrease for the velocity distribution enhances along the boundary layer as depicted in Figure 5. Also, from this figure, it is clear that with the increase of the porous parameter, the thermal boundary layer becomes thicker, but the momentum boundary layer becomes thinner.

The effect of the Eckert number $E_{c}$ on velocity and temperature profiles is shown in Figures 6 and 7, respectively. From Figure 6, we see that the velocity curve is lower when the Eckert number is larger, and so, the momentum effect is lower. Also, from Figure 7, we notice that the thermal boundary layer becomes thicker when the Eckert number increases, but the temperature distribution enhances. 
TABLE 1: Comparison of the present work with the published work.

\begin{tabular}{lcc}
\hline & Rajagopal et al. [24] & Present work \\
\hline 0.0 & 0.98561340 & 0.98561423 \\
1.0 & 0.27908819 & 0.27908734 \\
2.0 & 0.09291179 & 0.09291328 \\
3.0 & 0.03295374 & 0.03295452 \\
4.0 & 0.01196183 & 0.01196265 \\
\hline
\end{tabular}

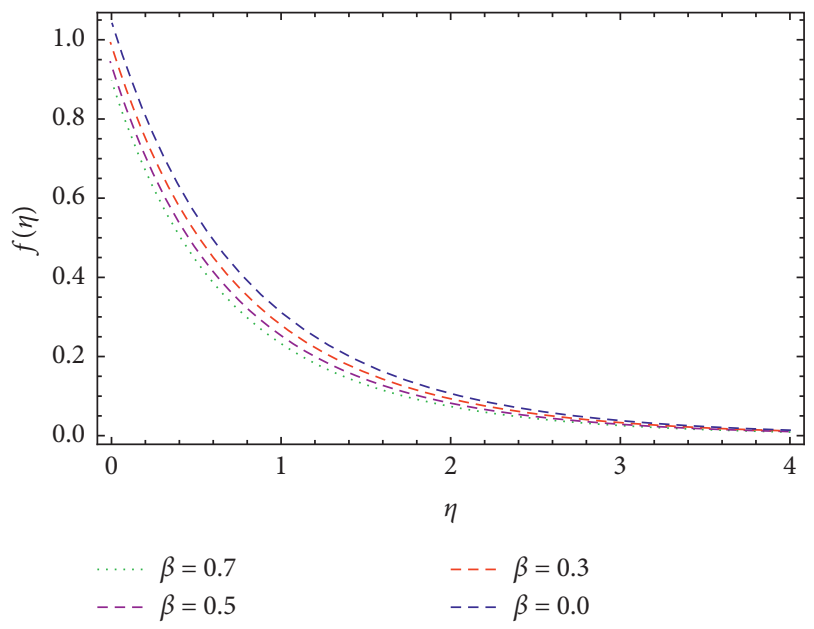

FIGURE 4: The influence of the velocity profile for different values of $\beta$, when $K=0.1, f_{w}=0.3, \lambda=0.2, \operatorname{Pr}=5.0, E_{c}=0.4$, and $a^{*}=$ $b^{*}=0.2$

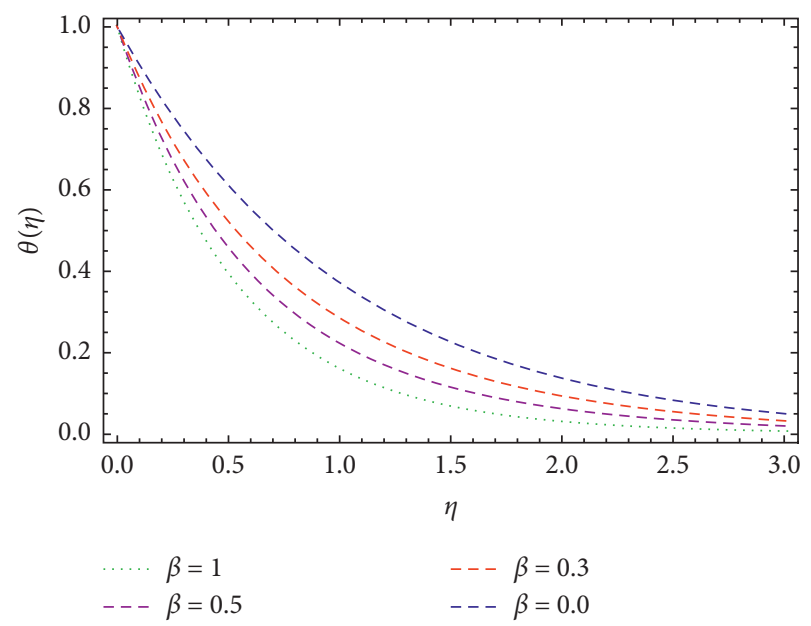

Figure 5: The influence of the temperature profile for different values of $\beta$, when $K=0.1, f_{w}=0.3, \lambda=0.2, \operatorname{Pr}=5.0, E_{c}=0.4$, and $a^{*}=b^{*}=0.2$.

Figures 8 and 9 are plotted to see the effect of the slip parameter versus similarity variable $\eta$ on velocity and temperature profiles. It is investigated that the velocity of the fluid decreases with the increasing values of the slip velocity parameter while with the increases of the same parameter, the temperature is increased.

The effect of the suction parameter $f_{w}$ on the fluid flow and temperature profile has been analyzed, and the results are given in Figures 10 and 11, respectively. These figures

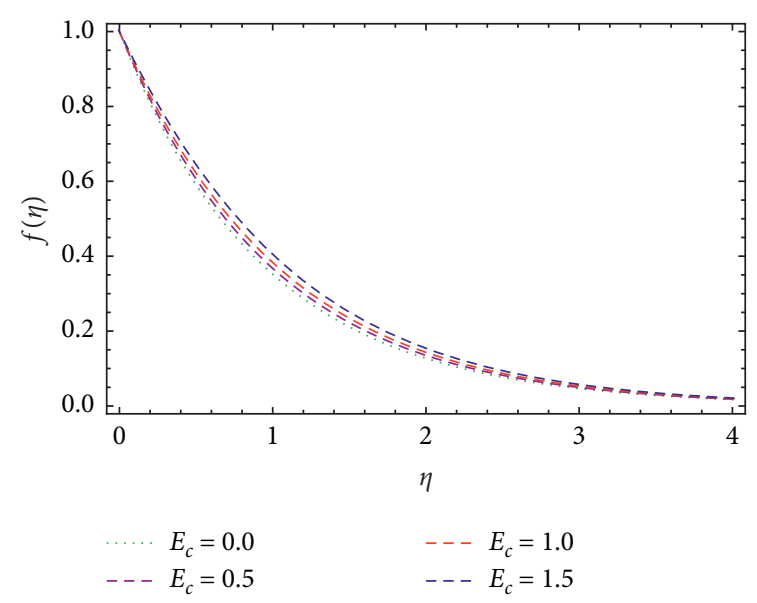

FIgURE 6: The influence of the velocity profile for different values of $E_{c}$, when $K=0.1, f_{w}=0.3, \lambda=0.2, \operatorname{Pr}=5.0, \beta=0.4$, and $a^{*}=$ $b^{*}=0.2$.

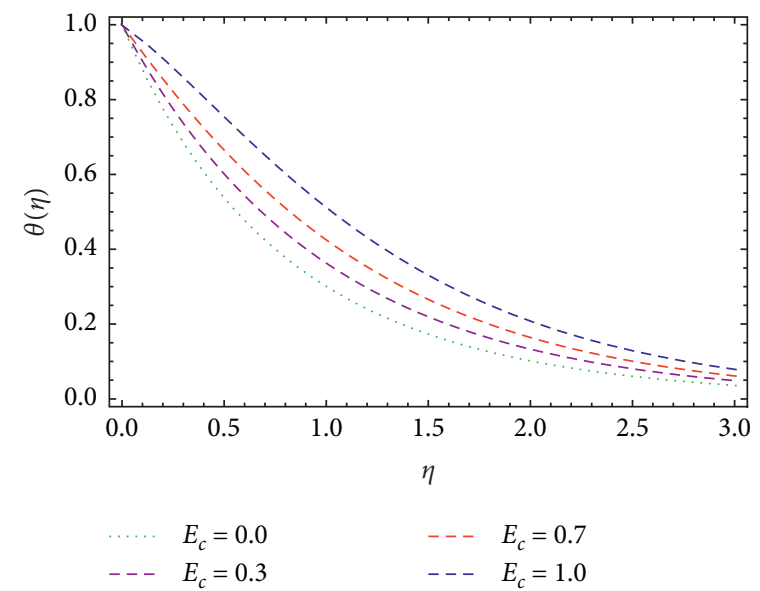

FIgURE 7: The influence of the temperature profile for different values of $E_{c}$, when $K=0.1, f_{w}=0.3, \lambda=0.2, \operatorname{Pr}=5.0, \beta=0.4$, and $a^{*}=b^{*}=0.2$.

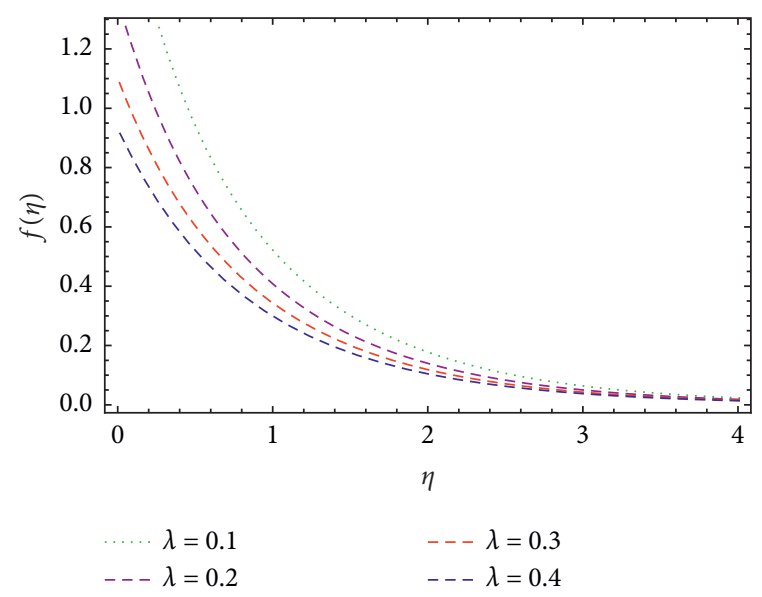

FIGURE 8: The influence of the velocity profile for different values of $\lambda$, when $K=0.1, f_{w}=0.3, \beta=0.2, \operatorname{Pr}=5.0, E_{c}=0.4$, and $a^{*}=$ $b^{*}=0.2$. 


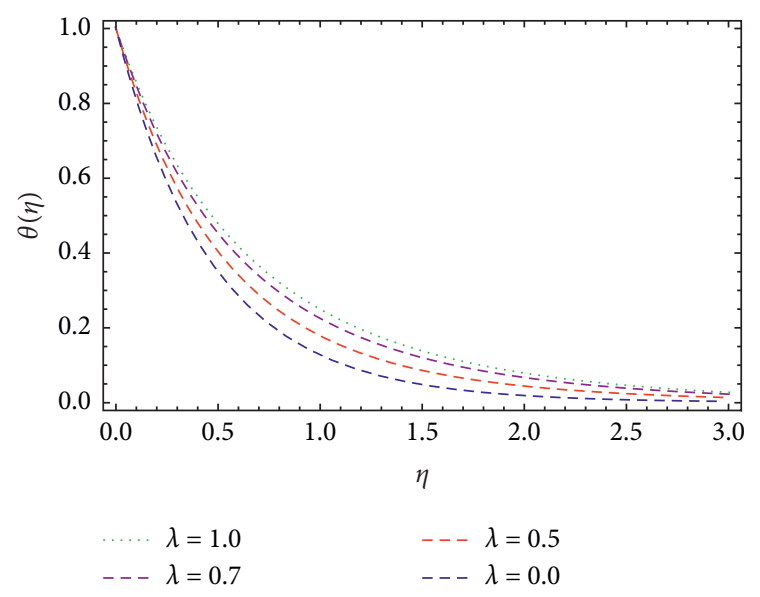

FIgURE 9: The influence of the temperature profile for different values of $\lambda$, when $K=0.1, f_{w}=0.3, \beta=0.2, \operatorname{Pr}=5.0, E_{c}=0.4$, and $a^{*}=b^{*}=0.2$.

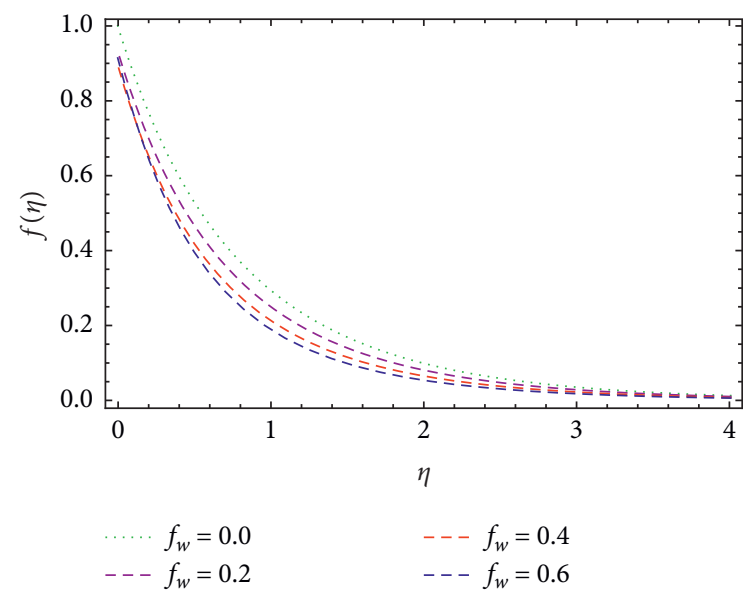

FIGURE 10: The influence of the velocity profile for different values of $f_{w}$, when $K=0.1, \beta=0.4, \operatorname{Pr}=5.0, E_{c}=0.4, \lambda=0.2$, and $a^{*}=$ $b^{*}=0.2$.

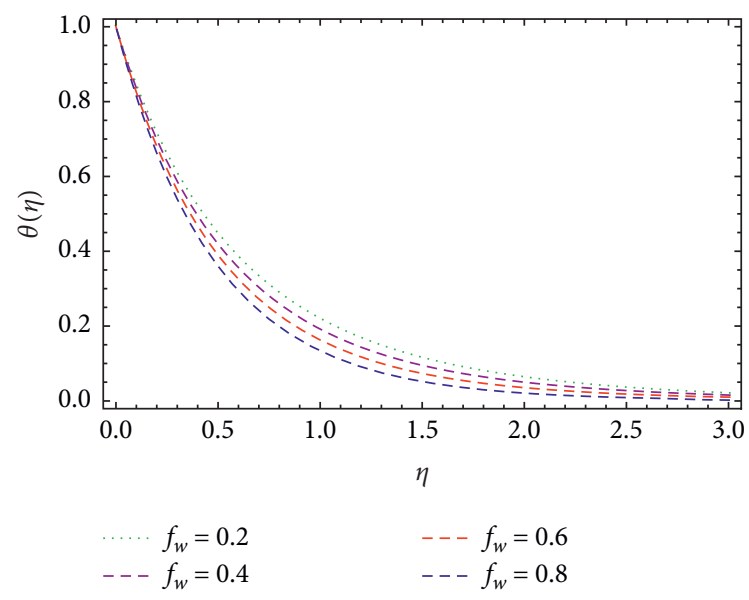

Figure 11: The influence of the temperature profile for different values of $f_{w}$, when $K=0.1, \beta=0.4, \operatorname{Pr}=5.0, E_{c}=0.4, \lambda=0.2$, and $a^{*}=b^{*}=0.2$.

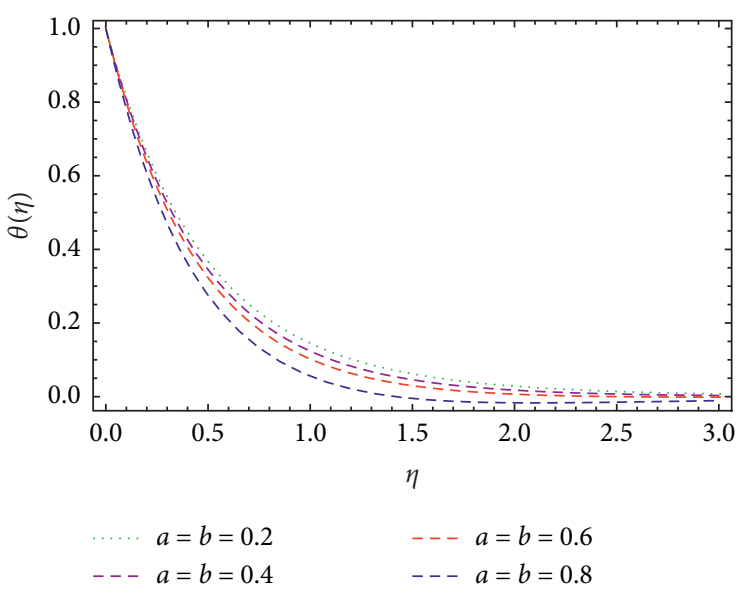

FIGURE 12: The influence of the velocity profile for different values of $a^{*}, b^{*}$, when $K=0.1, \beta=0.4, \operatorname{Pr}=5.0, E_{c}=0.4, \lambda=0.2$, and $f_{w}=0.3$.

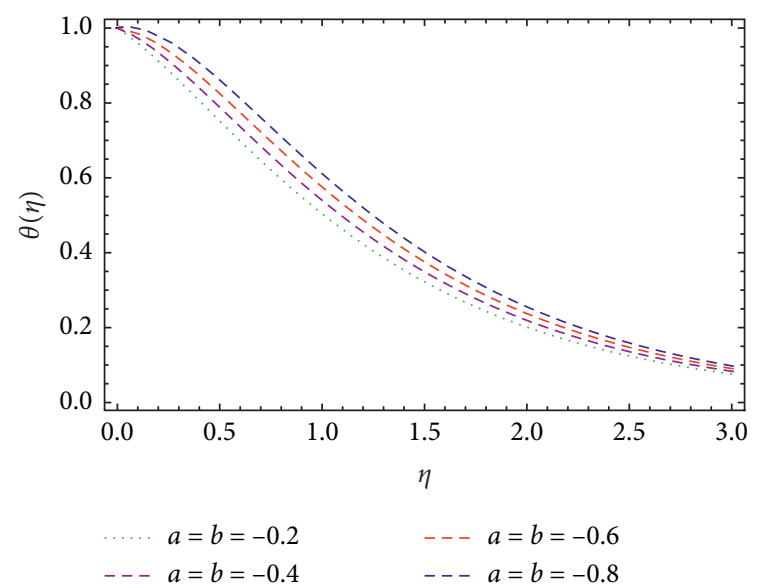

FIgURE 13: The influence of the temperature profile for different values of $a^{*}, b^{*}$, when $K=0.1, \beta=0.4, \operatorname{Pr}=5.0, E_{c}=0.4, \lambda=0.2$, and $f_{w}=0.3$.

show that the suction parameter has a significant effect on the boundary layer thickness. The suction parameter reduces the boundary layer thickness; as a result, the fluid flow and the temperature distribution reduce.

The effects of internal heat generation parameters on the thermal boundary layer thickness are presented in Figures 12 and 13. It is observed that as the values of the internal heat generation parameters $a>0$ and $b>0$ become stronger, the thermal boundary layer thickness increases, whereas the internal heat generation parameters $a>0$ and $b>0$ have the opposite effect. Also, it is noticed that the highest temperature distribution for the fluid in the boundary layer was obtained with the greatest heat generation parameters $a^{*}>0$ and $b^{*}>0$. Likewise, it is shown that the effect of the heat absorption parameters $a^{*}>0$ and $b^{*}>0$ causes a drop in the temperature distribution as the heat following from the sheet is absorbed.

At last for the accuracy of the problem, the present work is also compared with the published work reported by 
Rajagopal et al. [24] and an outstanding agreement was found and is also clarified in Table 1.

\section{Conclusions}

The homotopy analysis method is a seminumerical scheme applied for the solution of the proposed model problem of heat transfer phenomena in a viscoelastic fluid through a stretching sheet surface embedded in a porous medium with viscous dissipation of internal heat generation/absorption and slip velocity. Convergence analysis of the method is presented graphically. The effects of emerging parameters on the solution have been discussed in detail. It is observed that the suction parameter reduces the thickness of the boundary layer flow. Similarly, the porosity and slip parameters have the same effect on the thickness of the boundary layer flow as observed in the suction parameter. Also, the thermal boundary layer and temperature distribution increase with the increasing values of the Eckert number. Additionally, the present work is compared with the published work reported by Rajagopal et al. [24] for limiting cases and good agreement is found.

\section{Nomenclature}

$\begin{array}{ll}x, y: & \text { Velocity components } \\ \kappa: & \text { Thermal conductivity of the fluid } \\ E_{c}: & \text { Eckert number } \\ \rho: & \text { Fluid density } \\ T: & \text { Fluid temperature } \\ \lambda: & \text { Velocity slip parameter } \\ \mu: & \text { Fluid viscosity } \\ K: & \text { Porous parameter } \\ f_{w}: & \text { Suction velocity } \\ A_{,}: & \text {Constants } \\ q^{\prime \prime \prime}: & \text { Internal heat generation } \\ C_{f}: & \text { Skin friction } \\ \mu_{e}: & \text { Dynamics viscosity } \\ c_{p}: & \text { Specific heat } \\ N u: & \text { Nusselt number } \\ T_{w}: & \text { Sheet temperature } \\ a: & \text { Velocity slip factor } \\ R_{\mathrm{ex}}: & \text { Reynolds number } \\ T_{\infty}: & \text { Ambient temperature } \\ \eta: & \text { Similarity variable } \\ \beta: & \text { Porous parameter } \\ v_{w}: & \text { Suction velocity } \\ v: & \text { Kinematics viscosity } \\ a^{*}, b^{*}: & \text { Heat generation parameters } \\ u: & \text { Sheet velocity } \\ \operatorname{Pr}: & \text { Prandtl number. } \\ & \end{array}$

\section{Data Availability}

The data used to support the findings of this study are available from the corresponding author upon request.

\section{Conflicts of Interest}

The authors declare that they have no conflicts of interest.

\section{Acknowledgments}

This study was supported by King Saud University, Riyadh, Saudi Arabia (Project number RSP-2019/33).

\section{References}

[1] L. J. Crane, "Flow past a stretching plate," Zeitschrift für Angewandte Mathematik und Physik ZAMP, vol. 21, no. 4, pp. 645-647, 1970.

[2] J. Vleggar, "Laminar boundary- layer behavior on continuous accelerating surfaces," Chemical Engineering Science, vol. 32, no. 12, pp. 1517-1525, 1977.

[3] B. K. Dutta, P. Roy, and A. S. Gupta, "Temperature field in flow over a stretching sheet with uniform heat flux," International Communications in Heat and Mass Transfer, vol. 12, no. 1, pp. 89-94, 1985.

[4] I. Pop and T. Y. Na, "A note on MHD flow over a stretching permeable surface," Mechanics Research Communications, vol. 25, no. 3, pp. 263-269, 1988.

[5] M. E. Ali, "On thermal boundary layer on a power-law stretched surface with suction or injection," International Journal of Heat and Fluid Flow, vol. 16, no. 4, pp. 280-290, 1995.

[6] K. Vajravelu and D. Rollins, "Heat transfer in a viscoelastic fluid over a stretching sheet," Journal of Mathematical Analysis and Applications, vol. 158, no. 1, pp. 241-255, 1991.

[7] H. I. Andersson, "MHD flow of a viscoelastic fluid past a stretching surface," Acta Mechanica, vol. 95, no. 1-4, pp. 227-230, 1992.

[8] A. Subhas and P. Veena, "Visco-elastic fluid flow and heat transfer in a porous medium over a stretching sheet," International Journal of Non-Linear Mechanics, vol. 33, no. 3, pp. 531-540, 1998.

[9] E. Sanjayanand and S. K. Khan, "On heat and mass transfer in a viscoelastic boundary layer flow over an exponentially stretching sheet," International Journal of Thermal Sciences, vol. 45, no. 8, pp. 819-828, 2006.

[10] M. M. Nandeppanavar, M. S. Abel, and K. Vajravelu, "Flow and heat transfer characteristics of a viscoelastic fluid in a porous medium over an impermeable stretching sheet with viscous dissipation," International Journal of Heat and Mass Transfer, vol. 53, no. 21-22, pp. 4707-4713, 2010.

[11] C. L. M. H. Navier, "Mémoire sur les lois du mouvement des fluids," Memoires de l'Acaddmie des Sciences de l'Institut de France, vol. 6, pp. 389-416, 1823.

[12] P. A. Thompson and S. M. Troian, "A general boundary condition for liquid flow at solid surfaces," Nature, vol. 389, no. 6649, pp. 360-362, 1997.

[13] Z. Abbas, Y. Wang, T. Hayat, and M. Oberlack, "Slip effects and heat transfer analysis in a viscous fluid over an oscillatory stretching surface," International Journal for Numerical Methods in Fluids, vol. 59, no. 4, pp. 443-458, 2009.

[14] M. Turkyilmazoglu, "Multiple solutions of heat and mass transfer of MHD slip flow for the viscoelastic fluid over a stretching sheet," International Journal of Thermal Sciences, vol. 50, no. 11, pp. 2264-2276, 2011.

[15] L. L. Ferrás, A. M. Afonso, M. A. Alves, J. M. Nóbrega, O. S. Carneiro, and F. T. Pinho, "Slip flows of Newtonian and viscoelastic fluids in a 4:1 contraction," Journal of NonNewtonian Fluid Mechanics, vol. 214, pp. 28-37, 2014.

[16] K. D. Singh, "Effect of slip condition on viscoelastic MHD oscillatory forced convection flow in a vertical channel with 
heat radiation," International Journal of Applied Mechanics and Engineering, vol. 18, no. 4, pp. 1237-1248, 2013.

[17] D. S. Krishan, "MHD mixed convection visco-elastic slip flow through a porous medium in a vertical porous channel with thermal radiation," Kragujevac Journal of Science, vol. 35, pp. 27-40, 2013.

[18] K. Vajravelu, S. Sreenadh, and R. Saravana, "Influence of velocity slip and temperature jump conditions on the peristaltic flow of a Jeffrey fluid in contact with a Newtonian fluid," Applied Mathematics and Nonlinear Sciences, vol. 2, no. 2, pp. 429-442, 2017.

[19] Z. Khan, H. U. Rasheed, I. Tlili, I. Khan, and T. Abbas, "Runge-Kutta 4th-order method analysis for viscoelastic Oldroyd 8-constant fluid used as coating material for wire with temperature dependent viscosity," Scientific Reports, vol. 8, no. 1, 2018.

[20] K. V. Prasad, H. Vaidya, and K. Vajravelu, "MHD mixed convection heat transfer over a non-linear slender elastic sheet with variable fluid properties," Applied Mathematics and Nonlinear Sciences, vol. 2, no. 2, pp. 351-366, 2017.

[21] V. B. Awati, "Dirichlet series and analytical solutions of MHD viscous flow with suction / blowing," Applied Mathematics and Nonlinear Sciences, vol. 2, no. 2, pp. 341-350, 2017.

[22] R. Ahmad, A. Farooqi, J. Zhang, and N. Ali, "Steady flow of a power law fluid through a tapered non-symmetric stenotic tube," Applied Mathematics and Nonlinear Sciences, vol. 4, no. 1, pp. 255-266, 2019.

[23] Z. Khan, M. Khan, S. Islam et al., "Analysis of magnetohydrodynamics flow and heat transfer of a viscoelastic fluid through porous medium in wire coating analysis," Mathematics, vol. 5, no. 2, p. 27, 2017.

[24] K. R. Rajagopal, T. Y. Na, and A. S. Gupta, "Flow of a viscoelastic fluid over a stretching sheet," Rheologica Acta, vol. 23, no. 2, pp. 213-215, 1984.

[25] D. B. Ingham, I. Pop, and P. Cheng, "Combined free and forced convection in a porous medium between two vertical walls with viscous dissipation," Transport in Porous Media, vol. 5, no. 4, pp. 381-398, 1990.

[26] D. Rees and J. L. Lage, "The effect of thermal stratification of natural convection in a vertical porous insulation layer," International Journal of Heat and Mass Transfer, vol. 40, no. 1, pp. 111-121, 1996.

[27] P. M. Beckett, "Combined natural- and forced-convection between parallel vertical walls," SIAM Journal on Applied Mathematics, vol. 39, no. 2, pp. 372-384, 1980.

[28] P. M. Beckett and I. E. Friend, "Combined natural and forced convection between parallel walls: developing flow at higher Rayleigh numbers," International Journal of Heat and Mass Transfer, vol. 27, no. 4, pp. 611-621, 1984.

[29] A. J. Chamkha and A.-R. A. Khaled, "Similarity solutions for hydromagnetic simultaneous heat and mass transfer by natural convection from an inclined plate with internal heat generation or absorption," Heat and Mass Transfer, vol. 37, no. 2-3, pp. 117-123, 2001.

[30] S. Liao, "An analytic solution of unsteady boundary-layer flows caused by an impulsively stretching plate," Communications in Nonlinear Science and Numerical Simulation, vol. 11, no. 3, pp. 326-339, 2006.

[31] Z. Khan, H. U. Rasheed, T. A. Alkanhal, M. Ullah, I. Khan, and I. Tlili, "Effect of magnetic field and heat source on upperconvected-maxwell fluid in a porous channel," Open Physics, vol. 16, no. 1, pp. 917-928, 2018.

[32] Z. Khan, W. A. Khan, H. Ur Rasheed, I. Khan, and K. S. Nisar, "Melting flow in wire coating of a third grade fluid over a die using Reynolds' and vogel's models with non-linear thermal radiation and joule heating," Materials, vol. 12, no. 19, p. 3074, 2019.

[33] S.-J. Liao, "A new branch of solutions of boundary-layer flows over a permeable stretching plate," International Journal of Non-Linear Mechanics, vol. 42, no. 6, pp. 819-830, 2007.

[34] S. J. Liao, "Beyond perturbation: review on the basic ideas of homotopy analysis method and its application," Advances in Mechanical Engineering, vol. 38, pp. 1-34, 2008.

[35] S. Abbasbandy, "The application of homotopy analysis method to nonlinear equations arising in heat transfer," Physics Letters A, vol. 360, no. 1, pp. 109-113, 2006.

[36] S. Abbasbandy, "Homotopy analysis method for heat radiation equations," International Communications in Heat and Mass Transfer, vol. 34, no. 3, pp. 380-387, 2007.

[37] T. Hayat, M. Khan, and M. Ayub, "On the explicit analytic solutions of an Oldroyd 6-constant fluid," International Journal of Engineering Science, vol. 42, no. 2, pp. 123-135, 2004.

[38] T. Hayat, M. Khan, and S. Asghar, "Homotopy analysis of MHD flows of an Oldroyd 8-constant fluid," Acta Mechanica, vol. 168, no. 3-4, pp. 213-232, 2004.

[39] M. Khan, Z. Abbas, and T. Hayat, "Analytic solution for flow of Sisko fluid through a porous medium," Transport in Porous Media, vol. 71, no. 1, pp. 23-37, 2008.

[40] Z. Abbas, M. Sajid, and T. Hayat, "MHD boundary-layer flow of an upper-convected Maxwell fluid in a porous channel," Theoretical and Computational Fluid Dynamics, vol. 20, no. 4, pp. 229-238, 2006.

[41] Z. Khan, M. A. Khan, N. Siddiqui, M. Ullah, and Q. Shah, "Solution of magnetohydrodynamic flow and heat transfer of radiative viscoelastic fluid with temperature dependent viscosity in wire coating analysis," PLoS One, vol. 13, no. 3, Article ID e0194196, 2018. 


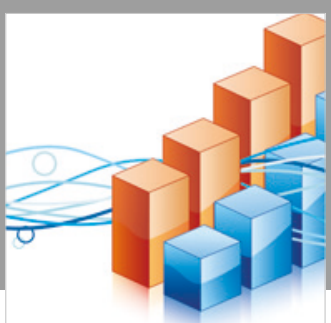

Advances in

Operations Research

\section{-n-m}
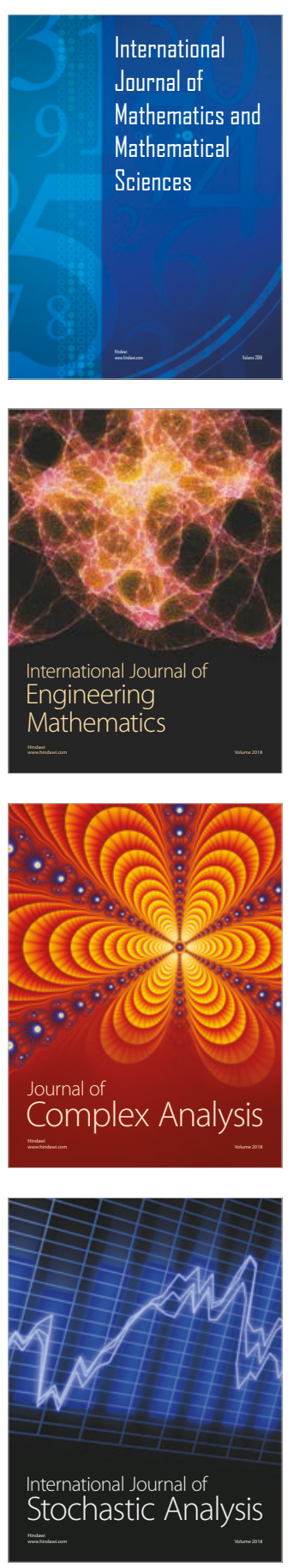
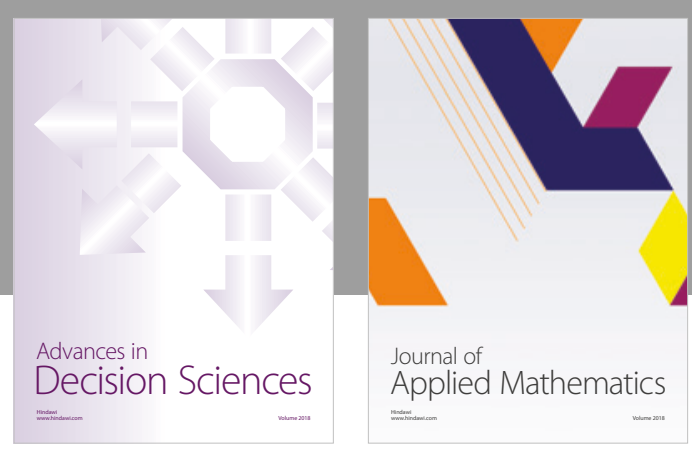

Journal of

Applied Mathematics
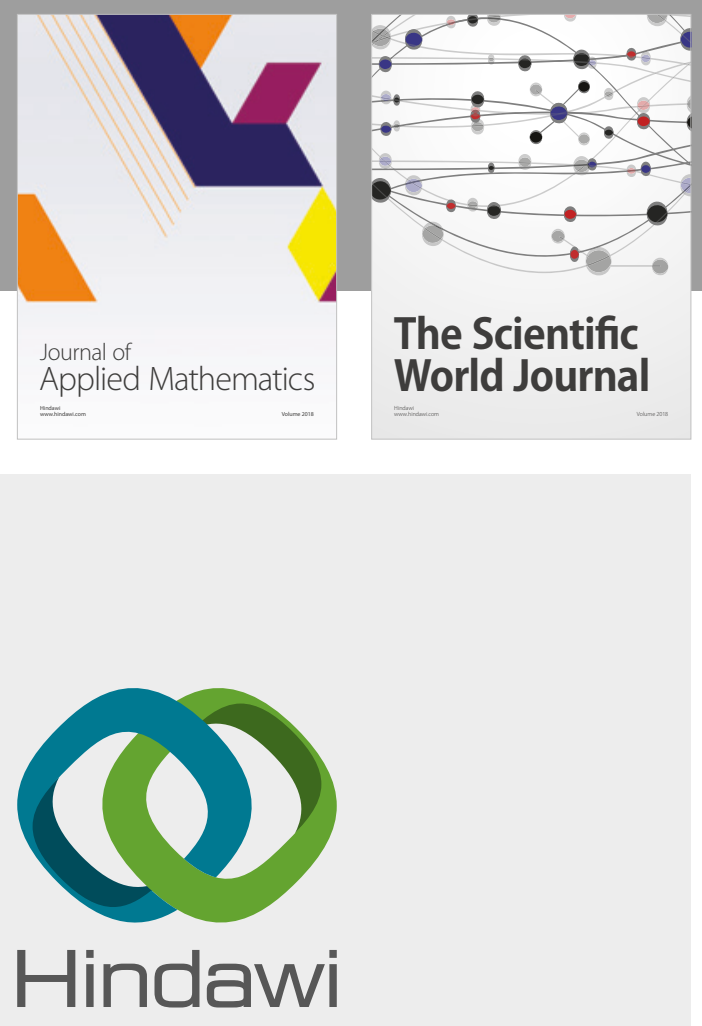

Submit your manuscripts at

www.hindawi.com

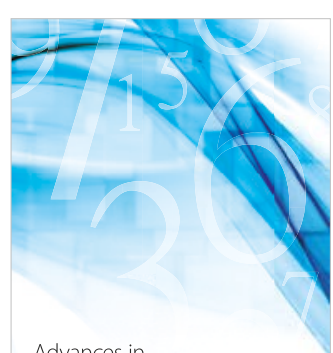

Advances in
Numerical Analysis
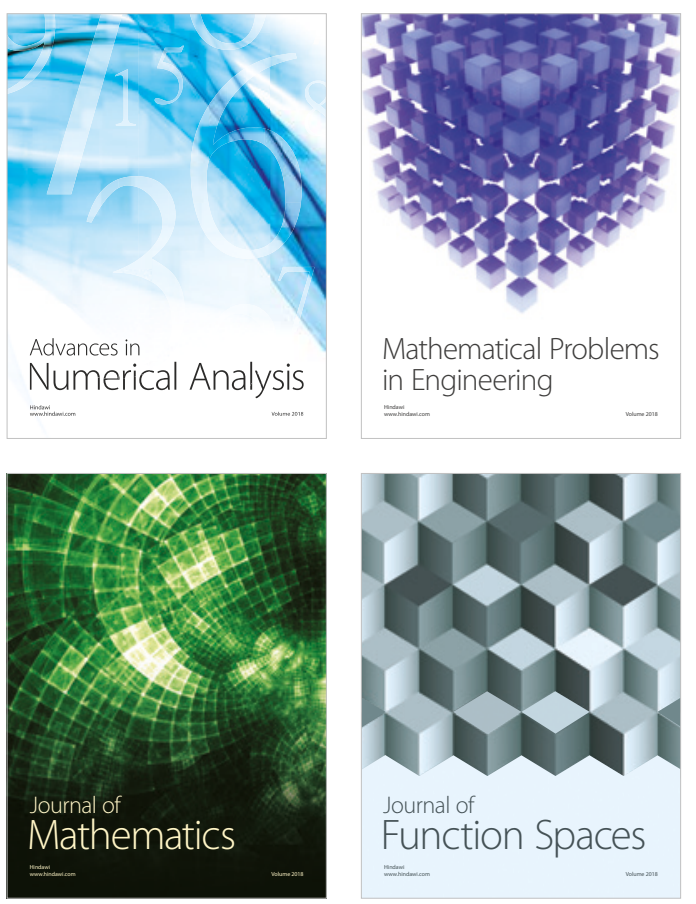

Mathematical Problems in Engineering

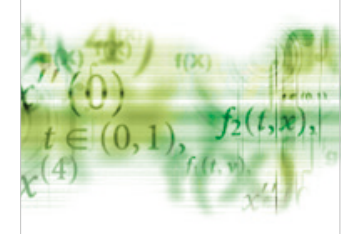

International Journal of

Differential Equations

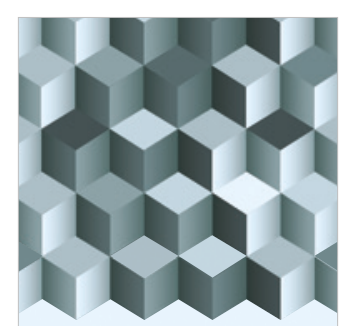

Journal of

Function Spaces
The Scientific

World Journal

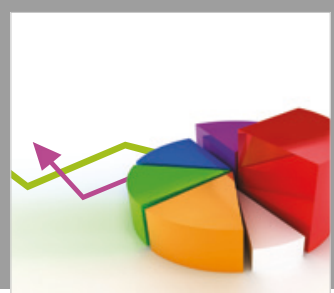

Journal of

Probability and Statistics
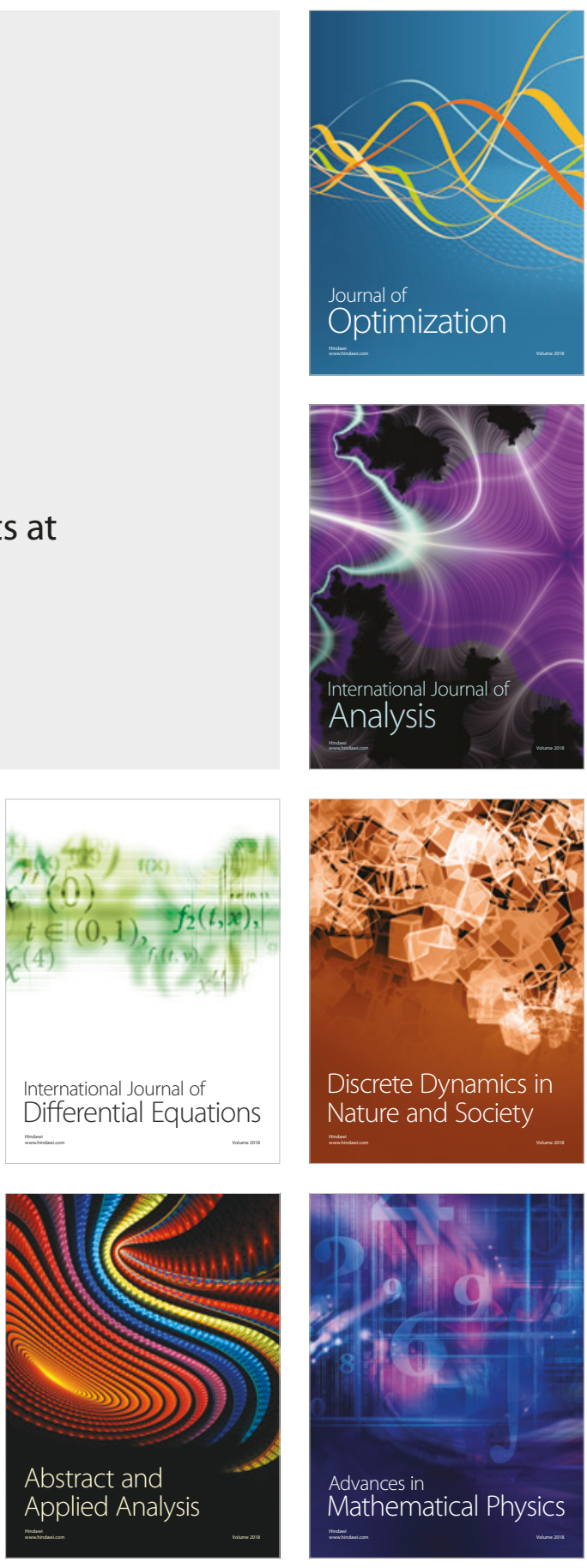